HUMANIDADES Y CIENCIAS SOCIALES

INVESTIGACIÓN

\title{
Producción de nucleoelectricidad y resistencias sociales: tres décadas de reclamos por una «Córdoba no nuclear»
}

Piaz, Agustín

\section{Resumen}

En este trabajo se estudian las acciones de resistencia a la tecnología nuclear en la provincia de Córdoba, Argentina, a partir de la descripción y el análisis de episodios contenciosos que emergen en torno al proceso productivo de la nucleoelectricidad desde el retorno a la democracia hasta la actualidad. Se identifican actores, argumentos y formas en que se implementa la resistencia al proceso productivo de la nucleoelectricidad, así como también las consecuencias y resultados que ha producido. Se sostiene que las acciones de resistencia a la tecnología nuclear en Córdoba se han desarrollado con diversos niveles de participación ciudadana y visibilidad en la esfera pública durante más tres décadas. Durante este período, han impulsado controversias por la producción de energía, la cuestión ambiental y promovido impactos sobre procesos tecnológicos y el hacer colectivo de reclamos.

Palabras clave: producción de nucleoelectricidad; resistencias sociales; protestas antinucleares; conflictos ambientales

Este artículo se inscribe en el marco de una investigación doctoral y posdoctoral (ambas realizadas con becas internas CONICET) sobre las acciones de resistencia a la tecnología nuclear en Argentina. Recibido el $21 / 10 / 2019$ y aceptado el 04/05/2020

DOI: https://doi.org/10.33255/3161/674

Autoría: LICH-CONICET-Universidad Nacional de San Martín (Argentina).

Contacto: apiaz@unsam.edu.ar 


\title{
Nuclear Power production and Social Resistances: Three decades of claims for an «anti-nuclear Córdoba»
}

\begin{abstract}
In this paper we study the resistance to nuclear technology in the Córdoba province of Argentina. We describe and analyze contentious episodes emerging since the return to democracy in 1983 over the nuclear power production cycle. The work identifies actors, arguments and contentious performances, as well as outcomes and consequences. We argue that resistance acts to nuclear technology in Córdoba have taken place with varied levels of citizen engagement and prominence in the public sphere for over three decades. During this period, resistance has prompted contention over power generation, environmental concerns and has promoted change to technological processes and collective claims.
\end{abstract}

Keywords: nuclear power; social resistances; antinuclear protests; environmental conflicts

\section{Produção de eletricidade nuclear e resistência social: três décadas de demandas por uma «Córdoba não nuclear»}

\section{Resumo}

Este trabalho estuda as ações de resistência à tecnologia nuclear na província de Córdoba, Argentina, a partir da descrição e análise de episódios contenciosos que emergem em torno do processo de produção da nucleoeletricidade desde o retorno à democracia até a atualidade. São identificados atores, argumentos e formas de implementação da resistência ao processo de produção da nucleoeletricidade, bem como as consequências e resultados que tem produzido. Argumenta-se que as ações de resistência à tecnologia nuclear em Córdoba têm sido desenvolvidas com diferentes níveis de participação cidadã e visibilidade na esfera pública por mais de três décadas. Durante esse período, elas têm impulsionado controvérsias sobre a produção de energia, a questão ambiental e promoveram impactos nos processos tecnológicos e na reivindicação coletiva.

Palavras-Chave: Produção de Nucleoeletricidade; Resistência Social; Protestos Antinucleares; Conflitos Ambientais 


\section{Introducción}

La provincia de Córdoba ha desempeñado un papel relevante en procesos de investigación y desarrollo de la tecnología nuclear en Argentina. Fue una de las primeras provincias en América Latina en solicitar un estudio de factibilidad para la instalación de una central nucleoeléctrica y en poner a disponibilidad de su universidad pública un reactor de investigación. Asimismo, fue pionera en la promulgación de legislaciones vinculadas con la política nuclear y en cuanto a la prospección y tratamiento del uranio, elemento utilizado como combustible tanto en reactores de investigación como de potencia (Martin, 2015). Enclave de la Regional Centro de la Comisión Nacional de Energía Atómica (CNEA), cuenta en su territorio con una central atómica de potencia, inaugurada en 1984 en la ciudad de Embalse; un reactor nuclear elemental (el RA-0), que se encuentra en la Universidad Nacional de Córdoba y es utilizado para investigación y formación de profesionales; el Complejo Minero-Fabril Los Gigantes (CMFLG); una planta productora de dióxido de uranio que forma parte del Complejo Fabril Córdoba (CFC); instalaciones con aplicaciones industriales y diversos centros de medicina nuclear. En este escenario, y en consonancia con el proceso de reapertura de la esfera pública para la participación ciudadana que comenzó tras el retorno a la democracia en 1983, emergieron desde los años ochenta y hasta la actualidad múltiples acciones de resistencia al proceso productivo de la nucleoelectricidad en el territorio provincial. Impulsadas por vecinos de zonas presuntamente afectadas y organizaciones no gubernamentales con marcado interés por la cuestión ambiental, se registraron durante las últimas tres décadas cuestionamientos y acciones de protesta contra: i) la extracción de uranio mediante minería a cielo abierto y los pasivos ambientales vinculados a estas actividades; ii) la producción de dióxido de uranio (material utilizado para la fabricación de combustibles nucleares) en el CFC; iii) la producción de energía en la central de Embalse; y iv) el manejo y disposición de combustibles gastados y residuos radioactivos en distintas instalaciones que funcionan bajo la órbita de la CNEA.

En este trabajo se estudian las acciones de resistencia a la tecnología nuclear en la provincia de Córdoba a partir de la descripción y el análisis de episodios contenciosos que emergieron en torno al proceso productivo de la nucleoelectricidad durante las últimas tres décadas. Más específicamente, por un lado, se identifican y caracterizan actores, argumentos y formas en que se implementó la resistencia, que en ocasiones derivó en acciones colectivas de protesta y procesos de discusión de la tecnología nuclear en la esfera pública; 
por otro lado, se describen y analizan impactos de la resistencia en procesos tecnológicos y el hacer colectivo de reclamos.

Este trabajo forma parte de una investigación más extensiva que se pregunta por las características y particularidades que presenta la resistencia a la tecnología nuclear en Argentina, uno de los tres únicos países de Latinoamérica -junto con Brasil y México- que cuentan con reactores de potencia y que se han erigido como líderes en cuanto al desarrollo y la implementación de esta tecnología en la región. Asimismo, y ante la identificada escasez de estudios sobre el tema, se lleva a cabo en el marco de: i) un renovado interés por la producción de nucleoelectricidad en el mundo, que se apoya en la caracterización de esta tecnología como una opción para enfrentar el calentamiento global (en tanto, según se busca destacar desde sectores promotores, la generación de energía mediante tecnología nuclear no genera gases que contribuyan con el efecto invernadero); ii) la puesta en marcha de Atucha II, los avances en la construcción de una Central Argentina de Elementos Modulares (CAREM) y los proyectos de construcción de nuevas centrales de potencia en el país impulsados mediante acuerdos alcanzados con China durante la presidencia de Cristina Fernández de Kirchner y ratificados durante la gestión de Mauricio Macri; y iii) de las nuevas protestas contra la producción de nucleoelectricidad que se registraron en el escenario global tras el accidente ocurrido en Fukushima en 2011, que reimpulsó acciones de resistencia a la tecnología nuclear, no siendo Argentina una excepción. En términos más generales, consideramos que este trabajo se inscribe en el marco de discusiones por la configuración de la matriz energética en Argentina ${ }^{1}$ y los modos pasibles de ser considerados ambientalmente sustentables para producir energía.

Los lineamientos teórico-metodológicos que guían la investigación articuLan literatura sobre controversias y resistencia a las tecnologías (Nelkin, 1995; Bauer, 2015) con literatura sobre movimientos sociales y política contenciosa (McAdam, Tarrow y Tilly, 2007; Tarrow, 2011), en sintonía con los crecientes esfuerzos que se han realizado desde el área de los Estudios Sociales de la Ciencia y la Tecnología por establecer diálogos y aportes recíprocos entre estos campos (ver Breyman et al., 2017). En este sentido, se busca prestar atención a los procesos de discusión por el desarrollo y la implementación de la tecnología nuclear que se dirimen en la esfera pública, así como las formas en que se expresa la resistencia -en tanto forma de participación política (Bauer, 2015: 50) que puede derivar en controversia- y los impactos que promueve. Asimismo, el trabajo se apoya en literatura sobre conflictividad ambiental y la disputa por la inequitativa distribución de riesgos y beneficios, caracterizada en términos de injusticia ambiental (Carruthers, 2008; Giugni y Grasso, 2015). 
La metodología es cualitativa, y se aborda el estudio de un caso conformado por acciones de protesta y cuestionamientos que emergieron en la provincia de Córdoba durante las últimas tres décadas hacia: los procesos extractivos del uranio en el CMFLG; la considerada inconveniente localización de planta productora de dióxido de uranio Dioxitek S.A.; los métodos de tratamiento de residuos radioactivos en el CFC; $y$ de las actividades vinculadas a la producción de nucleoelectricidad en la Central Atómica de Embalse. Consideramos, además, que se trata de un estudio representativo de las acciones de resistencia a la tecnología nuclear en Argentina, en tanto en el devenir de los episodios contenciosos participan actores, se sostienen argumentos y se ponen en juego repertorios de acción colectiva recurrentes en diversas acciones de protesta contra el proceso productivo de la nucleoelectricidad (ver Piaz, 2015, 2016, 2020).

Con el objetivo de recuperar y poner en escena la perspectiva de los actores que promueven y sostienen las acciones de resistencia a la tecnología nuclear en la provincia de Córdoba, se revisaron y analizaron fuentes primarias de información (comunicados institucionales, sitios web); se realizaron entrevistas en profundidad con miembros representativos de organizaciones de movimiento social y ONG como FUNAM, Greenpeace, Conciencia Solidaria, entre otros integrantes del Colectivo Córdoba No Nuclear; y se llevaron a cabo observaciones participantes en acciones colectivas de protesta en el territorio provincial. Asimismo, el trabajo de campo se complementó con la revisión y el seguimiento documental de los episodios contenciosos en medios de comunicación gráficos y audiovisuales. Si bien el foco analítico de la investigación se orienta hacia el polo conformado por los actores resistentes, se realizaron también entrevistas en profundidad a representantes de sectores promotores del desarrollo nuclear en Córdoba, como trabajadores y asesores de la CNEA, así como a funcionarios públicos que se desempeñaban en áreas gubernamentales vinculadas a la ciencia, la tecnología y la cuestión ambiental.

\section{Las primeras voces de oposición}

Tras el retorno a la democracia en 1983, en el marco de un proceso de reapertura de la esfera pública que favoreció la participación ciudadana, comenzaron a constituirse en Argentina múltiples organizaciones de la sociedad civil interesadas en diversas temáticas como los derechos humanos, acceso a la vivienda, cuestiones de género y la cuestión ambiental (Forni y Leite, 2006: 217-218). En Córdoba, la Fundación Nacional para la Defensa del Ambiente se destacó entre 
este último grupo conformado por noveles organizaciones ambientalistas que, junto con otros miembros de la Red Nacional de Acción Ecologistǎ-como el Movimiento Antinuclear del Chubut, la Sociedad Ecológica Regional y BIOS Argentina-, fueron pioneras en impulsar la resistencia al desarrollo nuclear en el país y cuestionar el proceso productivo de la nucleoelectricidad. Sin embargo, no fue sino hasta la segunda mitad de la década del ochenta que adquirieron notoriedad en Argentina las primeras voces de oposición al desarrollo y la implementación de esta tecnología en la esfera pública, en un escenario global caracterizado por uno de los picos de oposición a la tecnología nuclear alcanzado tras el accidente ocurrido en Chernóbil en 1986.

Mientras que en la Patagonia Argentina comenzó a desarrollarse una controversia -que se extendería por más de una década- en torno al proyecto de creación de un repositorio final de residuos radiactivos de alta actividad en la provincia de Chubut (Piaz, 2015; Dichdji, 2018), en la provincia de Córdoba comenzaron a ser cuestionados los procesos extractivos y de purificación del uranio. Más específicamente, miembros de la RENACE expresaron su preocupación respecto a las actividades extractivas de uranio que se llevaron a cabo en el CMFLG durante la década del ochenta, las cuales fueron asociadas a impactos negativos sobre el ambiente, como la destrucción de la flora y fauna nativa, y la presunta contaminación de ríos linderos al sitio popularmente conocido como Los Gigantes. Asimismo, surgieron también cuestionamientos a las actividades que se desarrollaban en el CFC y se vinculaban, por un lado, con el tratamiento y disposición de residuos radioactivos en el sitio conocido como «el chichón»-; por otro lado, con los procesos de producción de dióxido de uranio realizados por la empresa Dioxitek S.A. Tras el cese de actividades extractivas en el CMFLG en 19894 la resistencia al desarrollo nuclear se reorientó en Córdoba hacia las actividades que se llevaban a cabo en el CFC. En este escenario, la resistencia a la producción de dióxido de uranio y el manejo y disposición de residuos radioactivos de baja actividad ganó visibilidad en la esfera pública hacia finales de los años ochenta y se extendió en las décadas venideras. Durante este período, tales actividades comenzaron a ser percibidas como riesgosas para el ambiente y la salud de la personas por organizaciones ambientalistas y vecinos del predio de nueve hectáreas conocido con el nombre de «La Atómica». Sin embargo, la presencia y las actividades de la CNEA no eran novedosas en la zona.

Los orígenes del predio se remontan a mediados del siglo pasado, cuando la Dirección General de Fabricaciones Militares le cedió en 1952 a la CNEA un terreno para que comenzara a investigar en Córdoba acerca de los métodos de procesamiento y purificación del uranio, en el marco del cre- 
ciente interés por el desarrollo nuclear que se registró por aquel entonces en Argentina y otros países alrededor del mundo (Hurtado, 2014: 35-96). Allí se instaló la que fuera conocida desde sus inicios como Planta Córdoba, una planta piloto en la que se hicieron avances referentes a técnicas tanto de investigación y procesamiento de uranio como de diversos minerales que provenían desde distintos puntos del país. El predio, que había pertenecido con anterioridad a la Sociedad Mixta para la Industrialización del Cromo y sus Derivados (SOMICROM), se ubicaba en una por entonces periférica zona norte de la ciudad, en las inmediaciones de no más de un puñado de casas quinta (Martin, 2015: 23). Un par de décadas más tarde, mientras Argentina avanzaba hacia el dominio del proceso de producción nacional de elementos combustibles nucleares, se sumó al CFC una planta productora de dióxido de uranio.

La decisión de construir esta planta en una provincia de la región central del país respondía a que resultaba una posición estratégica, relativamente equidistante de los complejos minero-fabriles que se explotaban por aquel entonces. En 1980 comenzó el proceso de integración de la planta al CFC y en 1982 la instalación fue puesta en funcionamiento de manera exitosa. Fundamentalmente, la tarea que allí empezó a realizarse consistía en procesar químicamente el concentrado de uranio -la denominada «torta amarilla» o «yellow cake»- para obtener el dióxido de uranio, que sería luego enviado a la Fábrica de Elementos Combustibles Nucleares (emplazada en el Centro Atómico Ezeiza) para continuar con la fabricación del combustible que sería finalmente utilizado para abastecer a las centrales de potencia y el creciente de número de reactores de investigación y producción de radioisótopos que funcionaban en el país.

Ahora bien, hacia mediados de los años ochenta el CFC ya no se ubicaba en las periferias de la ciudad. Por el contrario, debido a la expansión demográfica impulsada por el incremento poblacional en la ciudad capital de Córdoba, el predio se encontraba rodeado por un creciente número de viviendas, situación que no se modificó hasta la actualidad. En suma: por un lado, crecimiento demográfico en las inmediaciones de un predio que formaba parte del proceso productivo de la nucleoelectricidad; por otro lado, la emergencia de las primeras resistencias al desarrollo nuclear en Argentina, en el marco de uno de los picos máximos de oposición a esta tecnología en el contexto global tras el accidente de Chernóbil en 1986.

En este escenario, las actividades de producción de dióxido de uranio y el manejo de residuos radioactivos comenzaron a ser percibidas como riesgosas para la salud y el ambiente por vecinos y organizaciones de la sociedad civil. 
De acuerdo con un representante de la FUNAM, la luz de alarma se encendió durante los años ochenta por la cercanía de la instalación a múltiples viviendas y, especialmente, porque «el nuclear es el peor de todos los problemas. No hay ninguna tecnología que pueda extender sus riegos por 2.400 siglos. No hay nada comparable» (Entrevistado A, entrevista personal, 21 de mayo de 2015). Asimismo, los cuestionamientos que problematizaban la ubicación del CFC en medio de un barrio residencial se potenciaron en el marco de las crecientes discusiones promovidas desde los estudios sobre urbanismo, que buscaban impulsar el ordenamiento del territorio y la regulación de los usos del suelo en zonas rurales y urbanas. Más específicamente, la Ordenanza Municipal n. ${ }^{\circ} 8.133$, sancionada en noviembre de 1985 , ponía en escena incompatibilidades en relación con la ubicación del CFC y las actividades allí realizadas 5 .

Hacia mediados de los noventa, los reclamos por la ubicación de Dioxitek continuaron incrementándose, sumándose a estos la emergencia de presunciones elaboradas por vecinos del CFC que comenzaron a vincular las actividades industriales allí realizadas con afecciones a la salud, principalmente cáncer. Aunque lejos de la masividad, la resistencia ganó visibilidad pública e impactó en la decisión del Municipio de Córdoba de solicitar en 1994 a las autoridades de la CNEA que revieran la localización de sus instalaciones y evaluaran un posible traslado. En noviembre de 1995 la CNEA y la Municipalidad de Córdoba coincidieron acerca de la inconveniente ubicación del CFC, accedieron a la suscripción de un convenio que establecía la conformación de una comisión para la reconversión del predio y acordaron su futura liberación luego de que se realizaran acciones y estudios necesarios. Sin embargo, eso no ocurriría, al menos, en un futuro inmediato.

\section{Intentos de relocalización y nuevos cuestionamientos}

En 1998 Dioxitek ratificó el compromiso de cese de actividades para ese mismo año, junto con la promesa de traslado de la planta y la remediación de pasivos ambientales vinculados al CFC, hecho que impulsó discusiones entre resistentes y promotores del desarrollo nuclear acerca de los métodos de tratamiento de los residuos radiactivos ubicados en el predio. Mientras que miembros de la FUNAM alertaban acerca de la existencia de «residuos radioactivos peligrosos», representantes de la CNEA buscaron relativizar las advertencias argumentando que estos residuos se encontraban «debidamente protegidos y almacenados [y que] no pueden considerarse peligrosos en los términos en que se considera el riesgo nuclear» (Pisani, 1997). En cuanto al traslado 
de Dioxitek, la localidad cordobesa de Despeñaderos resultó preseleccionada debido a facilidades acceso y ubicación (se encuentra a aproximadamente cincuenta kilómetros de la capital provincial) .

A comienzos de 1999 la CNEA avanzó en las negociaciones con las autoridades locales y la compra del predio se produjo en abril de ese mismo año. Sin embargo, cuando tomó estado público la iniciativa, vecinos de la zona que habían sido alertados y contaban con el apoyo de organizaciones ambientalistas como FUNAM y la ONG internacional Greenpeace, que se involucraba cada vez más en la lucha antinuclear en Argentina, comenzaron a movilizarse y convocar asambleas para discutir acerca de la conveniencia de la radicación de la planta en su territorio. Las preocupaciones se expresaron desde diversos sectores de la sociedad, entre los que se destacaron asociaciones vecinales y cooperativas agrícolas, en tanto una de las principales actividades comerciales de la zona es la producción agropecuaria, entre las que se destaca el cultivo de soja. La amenaza percibida en relación con una de las fuentes principales de ingresos resultó clave para dar visibilidad al conflicto en la esfera pública local e impulsar acciones colectivas de protesta. Luego de reiteradas manifestaciones de descontento -entre las que se destacó, según organizaciones ambientalistas, el «primer tractorazo antinuclear» («El traslado de la planta de uranio divide a Despeñaderos», 1999)-, la relocalización de Dioxitek en Despeñaderos fue desestimada y la provincia de Mendoza se presentó como la alternativa con mayores posibilidades.

Los argumentos para impulsar esta opción ponían el acento en la considerada conveniencia en cuanto a términos operativos de instalar la planta productora de dióxido de uranio en las inmediaciones del Complejo Minero Fabril Sierra Pintada y a la existente vinculación entre la planta y la provincia de Mendoza, propietaria del $1 \%$ de las acciones de Dioxitek S.A. Los planes de traslado se asociaron, a su vez, con la proyección de reapertura de la mina de Sierra Pintada y, en términos más generales, con la proyección de la reactivación de la minería de uranio en el país. Como parte de este plan, la CNEA había solicitado un estudio de prefactibilidad para comenzar nuevamente con los procesos extractivos de uranio (Martínez Demarco, 2012). Sin embargo, los planes de reapertura de la mina y la posibilidad de traslado de la planta alertaron a pobladores y organizaciones ambientalistas, que expresaron públicamente su rechazo.

Haciendo lugar a los reclamos, el intendente de San Rafael sostenía que se trataba de «una decisión que no puede tomar solo el intendente o las autoridades del departamento. Aquí debe involucrarse y opinar la comunidad» (Felix, O., citado en «Polémica por posible traslado de una planta atómica a 
San Rafael», 2004, párr. 18). En el marco de presentaciones judiciales realizadas por organizaciones ambientalistas $\underline{-}$ para impedir la reapertura del Complejo Minero San Rafael, la Corte Suprema de Justicia de Mendoza ordenó a la CNEA remediar pasivos ambientales resultantes de actividades pasadas. En tanto el proceso de restauración ambiental estaba lejos de concretarse ${ }^{8}$, el proyecto de relocalización en Mendoza no prosperó. Asimismo, circulación de información y los lazos que se establecieron entre actores resistentes impactaron en la reactivación de pedidos por el cierre de Dioxitek en la capital cordobesa, en consonancia con resistencias varias al desarrollo nuclear que habían comenzado a ganar visibilidad pública a finales de la década del noventa y principios del nuevo milenio.

\section{La resistencia en el nuevo milenio}

Hacia finales de la década del noventa se registró en varias provincias argentinas la emergencia de al menos tres controversias en torno al manejo y/o disposición final de residuos radioactivos, una de las dimensiones más cuestionadas y resistidas del proceso productivo de nucleoelectricidad. Entre estas se encontraban aquellas conformadas por: i) las movilizaciones masivas contra la instalación de un repositorio final de residuos radiactivos de alta actividad en la localidad patagónica de Gastre, en la provincia de Chubut, que impactarían en la desestimación del proyecto oficializada por la CNEA en 1997; ii) las discusiones en torno a la venta de un reactor nuclear de investigación a Australia por parte de la empresa INVAP S.A., y la posibilidad de que ingresara al país combustible gastado o -en términos de los actores resistentes- desechos radioactivos, para ser acondicionados en la Argentina; y iii) cuestionamientos a los métodos de tratamiento de desechos radioactivos líquidos implementados en el $C A E$, que tomaron estado público en el año 2000, e impulsaron la apertura de una causa judicial y acciones de protesta varias (ver Piaz y Vara, 2013; Piaz, 2015).

En un escenario signado además por el surgimiento de múltiples conflictos ambientales en el país y la región?, los cuestionamientos a la considerada inconveniente localización de Dioxitek, el manejo de residuos radioactivos en el CFC y el saneamiento de pasivos ambientales resultantes de las actividades mineras en Córdoba continuaron desarrollándose y alcanzaron un nuevo estadio tras la reactivación del Plan Nuclear Argentino en 2006 y el accidente nuclear ocurrido en 2011 en la ciudad japonesa de Fukushima Daiichi. Por un lado, la reactivación del PNA en 2006 dio un nuevo impulso al desarrollo nuclear en Argentina, al tiempo que potenció el estado de alerta entre históricos y más recientes actores antinucleares ante la proyectada reactivación 
de actividades vinculadas con el proceso productivo de la nucleoelectricidad, como la minería de uranio o la construcción de nuevos reactores de potencia. Por otro lado, el accidente ocurrido en Fukushima -luego de que un terremoto y un tsunami azotaran la costa japonesa, provocando fallas en los sistemas de refrigeración de los reactores y la consecuente fusión parcial de los núcleos- volvió a poner a la tecnología nuclear en el centro de la atención de movimientos ambientalistas, la agenda pública y los medios de comunicación masiva, impactando en la reinstalación de controversias en el país $\underline{\underline{10}}$.

En el marco de este proceso reconocido e interpretado como una oportunidad para impulsar las discusiones en torno a la producción de nucleoelectricidad en la esfera pública e instalar el debate en la agenda política y mediática a nivel nacional, organizaciones vecinales y ambientalistas de la provincia de Córdoba establecieron acuerdos y acciones de trabajo conjunto que promovieron la resistencia al desarrollo nuclear a partir de la construcción de un espacio colectivo que autodenominarían Plataforma Córdoba No Nuclear. Bajo la premisa de «fomentar la participación ciudadana y exigir que Córdoba abandone la energía atómica» (Córdoba No Nuclear, s/f), múltiples organizaciones -entre las que se encontraban la ONG Greenpeace, la Fundación CEDHA, el portal de medio ambiente y ecología Eco-Sitio, Los Verdes-FEP, la Fundación Tierravida, la Fundación Holderín y la Fundación para el Desarrollo de Políticas Sustentables (FUNDEPS)프- aunaron esfuerzos para impulsar la resistencia antinuclear. De este modo, los actores resistentes pusieron en juego al menos tres mecanismos que McAdam, Tarrow y Tilly (2007) identifican como relevantes en los episodios contenciosos y que han denominado «brokerage», «identity shift» $y$ «diffusion».

En relación con la noción de «brokerage», que los autores utilizan para referirse a las nuevas conexiones entre grupos -o bien no conectados previamente, o con conexiones previas pero no tan estrechas-, se observa que si bien las organizaciones que participarían del colectivo Córdoba No Nuclear contaban previamente con un interés común por la cuestión ambiental, los posicionamientos compartidos en contra del proceso productivo de la nucleoelectricidad resultaron claves para establecer nuevos vínculos y/o reforzar vínculos previos. Asimismo, al participar de un flamante colectivo de actores se observa también cómo las organizaciones integrantes -aunque sea de manera temporal- se presentan conjuntamente en el marco de una nueva identidad colectiva, implementando un mecanismo que desde la teoría de los estudios sobre política contenciosa se ha caracterizado como «identity shift».

En cuanto a la forma de llevar adelante los reclamos en el espacio público, se observa que mediante la realización de performances (como la instalación de un teléfono rojo en la plaza principal de la ciudad), la divulgación de infor- 
mación -en espacios como foros y asambleas vecinales, charlas públicas- y la concreción de acciones de protesta en inmediaciones de las instalaciones rechazadas (como la irrupción en la central de Embalse y el despliegue de banderas en el edificio del reactor), se buscó impulsar la resistencia al desarrollo nuclear y dar visibilidad a la controversia en la esfera pública. En sintonía con la caracterización que Svampa (2012: 97-101) propone para los movimientos socioambientales en la región, las organizaciones agrupadas bajo el lema «Córdoba No Nuclear» priorizaron la acción directa, el impulso de demandas legales (como se mostrará a continuación) y la conformación de estructuras de participación no jerárquicas que propician la toma de decisiones compartidas y democráticas, poniendo en juego así el mecanismo denominado en términos de «diffusion».

Ahora bien, en aquel momento los reclamos no se cernían a pedidos por el cierre y/o relocalización de Dioxitek y el saneamiento del CFC. Recuperaban, en cambio, pedidos por la remediación ambiental del CMFLG y cuestionaban la extensión de vida de la central nuclear Embalse, cuyo ciclo de actividad inicialmente programado había concluido. Es decir, no se trataba de la emergencia de reclamos aislados hacia una instalación nuclear o una problemática en particular, sino de cuestionamientos consensuados y consolidados entre actores resistentes que se habían vuelto extensivos al proceso productivo de la nucleoelectricidad. En este sentido se expresaban también asambleas barriales y organizaciones ambientalistas con base en distintos puntos del territorio nacional. Mediante un comunicado de prensa titulado «Córdoba frente a los peligros de la energía nuclear» difundido en mayo de 2011 , miembros de la RENACE destacaron riesgos asociados a la actividad nuclear y consideraron fundamental un debate acerca de los modos de producir energía y, en términos más generales, sobre cómo pensar y problematizar la cuestión ambiental:

El terrible accidente de Fukushima motivó inmediatos análisis sobre el uso de la energía nuclear. Decenas de países revisan sus planes nucleares, se dan manifestaciones multitudinarias [...]. Córdoba tiene todos los peligros nucleares posibles: Contaminación radiactiva actual [...], un basurero nuclear en plena ciudad capital, una central con repetidas fallas y el entramado de ríos en peligro frente a futuras extracciones de uranio. [...] ¿Cómo solucionar los problemas del presente y evitar más daños a futuro? Creemos que debe debatirse, en el marco de la crisis energética global, que al igual que la financiera, la alimentaria y la climática, nos exige revisar nuestro modo de vida y pasar a acciones concretas de transición para salvarnos de colapsos y sufrimientos. («Córdoba frente a los peligros de la energía nuclear», 2011, párr. 1-4) 
Del comunicado se desprende que, mientras la mención a la «contaminación radioactiva» y al «basurero nuclear» remite a las actividades de producción de dióxido de uranio y la disposición transitoria de material radioactivo en el CFC, se alerta también acerca de peligros percibidos en torno al funcionamiento de Embalse y los pasivos de la minería de uranio en el CMFLG. En el marco de este período de auge y visibilidad que adquirió la resistencia al desarrollo nuclear, la Municipalidad de Córdoba avanzó en septiembre de 2012 hacia la clausura de Dioxitek. Entre los argumentos sostenidos por las autoridades para clausurar la planta se explicitó la violación de la ordenanza del uso de suelo que regía en la capital provincial desde hacía aproximadamente dos décadas y la caducidad de los acuerdos alcanzados para su relocalización $n^{12}$.

Como contrapartida, hacia finales de ese mismo año las autoridades de Dioxitek elevaron a la justicia un pedido de amparo para que se les permitiera continuar funcionando y no poner en riesgo, de acuerdo con su interpretación, el abastecimiento de combustible nuclear. Durante el mes de octubre el Juzgado Federal n. 1 hizo lugar a la demanda, dejando sin efecto la sanción municipal. De manera simultánea la Municipalidad de Córdoba y la empresa acordaron un nuevo plazo para efectivizar el cese de actividades y proceder con el traslado de la planta. Asimismo, se acordó avanzar hacia la remediación de los pasivos ambientales identificados, especialmente aquellos vinculados al sitio «el chichón». Mientras se establecía otra promesa para el cierre de Dioxitek, nuevos (y reiterados) destinos comenzaron a barajarse.

\section{Nuevos horizontes, nuevas negativas}

La provincia de La Rioja -en la región noroeste de Argentina, en la zona cordillerana limítrofe con Chile- comenzó a barajarse como posible destino para Dioxitek ${ }^{\underline{13}}$. En el marco de crecientes resistencias a la proyección de actividades vinculadas con la minería de uranio en la provincia, el rechazo por parte de organizaciones ambientalistas y asambleas vecinales al emplazamiento de la planta no tardó en llegar. La Asamblea Riojana Capital, perteneciente a la región Cuyo-NOA de la Unión de Asambleas Ciudadanas, se posicionó desde un primer momento como uno de los grupos que participarían de manera más activa de las por entonces incipientes discusiones y acciones de protesta, que incluyeron desde charlas entre vecinos hasta acampes, manifestaciones y -en ocasiones más extremas- bloqueos de rutas. Incluso, de acuerdo con lo sostenido por la Asamblea, sus acciones han impactado de manera directa en la elaboración y sanción de la Ordenanza Municipal n. ${ }^{\circ} 4.930$, ocurrida en agosto de 2012. Entre otras cuestiones, esta ordenanza proclama la capital 
provincial como municipio «no tóxico, no nuclear y ambientalmente sustentable»14. En el marco de reiteradas protestas, las negociaciones por el traslado de la planta productora de dióxido de uranio finalmente no avanzaron.

Mientras la opción por La Rioja perdía terreno, a comienzos de 2013 se conocieron las intenciones del municipio de Río Tercero -ubicado aproximadamente a unos ochenta kilómetros de la ciudad capital de Córdoba- de analizar un posible traslado de Dioxitek. Como respuesta a la iniciativa, pobladores locales con el apoyo de organizaciones ambientalistas protagonistas de la lucha antinuclear expresaron públicamente sus preocupaciones en redes sociales y medios de comunicación masiva, como la prensa gráfica, radial y televisiva. Uno de los argumentos centrales hacía referencia a la prohibición de instalaciones nucleares según lo estipulado en la normativa local. Desde 2007, el artículo 55 de la Carta Orgánica de la ciudad de Río Tercero declara al territorio de la jurisdicción municipal como «zona protegida» $\underline{15}$.

La existencia de esta normativa no solo potenció la controversia sino que también reorientó las discusiones hacia la interpretación de aspectos jurídico-técnicos. Por un lado, surgieron interpretaciones divergentes acerca de cuáles son aquellas industrias que pueden ser consideradas nucleares $\frac{16}{6}$. Por otro lado, se potenciaron discusiones acerca de los parámetros que posibilitan señalar la existencia de peligros para la salud de las personas y el ambiente, en tanto la normativa prohíbe la «manipulación, uso y/o transporte de sustancias o insumos nucleares o afines, que pongan en peligro la vida, la salud de los habitantes y el ecosistema». Mientras que para los actores resistentes Dioxitek era una planta vinculada con actividades nucleares y que representaba potenciales riesgos, para el intendente su instalación no pondría nada en peligro: «El texto [la carta orgánica municipal] dice que no se permiten esas industrias, que pongan en peligro la vida. Pero esta empresa no pondrá en peligro nada» (citado en Mongi y Martínez, 2013).

En este escenario, directivos de la empresa decidieron continuar con el sondeo de otros destinos para la relocalización de la planta. Un predio lindero a la central de Embalse -también a unos aproximadamente ochenta kilómetros de distancia de la ciudad capital, en el Valle de Calamuchita- fue el escogido. Para justificar la elección se destacó la existencia previa de actividades nucleares en el municipio, únicas de su tipo en la provincia de Córdoba. Para mediados de 2013, el intendente de Embalse había aprobado los estudios correspondientes de factibilidad para la instalación de la planta. Según el dirigente, su decisión se fundamentaba en la existencia de «una cuestión muy lógica: los únicos en esta provincia que tenemos actividad nuclear somos los embalseños, salvo lo que hoy produce Dioxitek en Córdoba» (citado por 
Mongi y Martínez, 2013: párr.9). En este sentido, sostenía además que en caso de darse una discusión pública, no debería centrarse en la instalación de la planta productora de dióxido de uranio sino más bien en el desarrollo y la implementación de la tecnología nuclear en términos más generales: «No queremos ya discutir Dioxitek, tenemos que discutir si queremos la actividad nuclear o no, pero con un debate serio, sin fundamentalismos» (ibidem).

La invitación al debate no sería desestimada por el colectivo Córdoba No Nuclear y organizaciones ambientalistas que históricamente se han opuesto al desarrollo de esta tecnología. Para aquel entonces, los originales pedidos de relocalización de Dioxitek habían sido desestimados: se habían reconvertido, en cambio, en reclamos por el cese definitivo de la planta. Tal como se expresa en una relectura de la problemática por parte de un referente de la agrupación ambientalista Los Verdes, los reclamos no deberían ser entendidos en términos restrictivos a una disputa por localización o NIMBY (acrónimo del inglés Not In My Back Yard); es decir, «no en mi patio trasero», sino como parte de una discusión acerca del proceso productivo de la nucleoelectricidad:

La cuestión de Dioxitek es mucho más profunda que su localización y relocalización. Dioxitek es parte del ciclo del combustible nuclear, y al hablar de esta industria no podemos pensarla de forma aislada sino en su totalidad, desde la extracción del uranio, pasando por su procesamiento, utilización en los reactores nucleares y su disposición luego de su uso. Ese debate es el que está faltando a nivel político y se tiene que dar en todos los niveles del Estado, pero especialmente a nivel nacional. Un buen porcentaje de la población lo está requiriendo, especialmente en aquellos lugares que se ven o se verán afectados por alguna de las fases de este proceso (Entrevistado C, entrevista personal, 21 de mayo de 2015).

Del comentario del Entrevistado C se desprende, al menos, la existencia de un doble desplazamiento. Por un lado, se produce un reenmarcado de las discusiones, a partir de la puesta en juego de un framing alternativo y más amplio que cuestiona a la totalidad del proceso productivo de la nucleoelectricidad. Por otro lado, se produce también un «cambio de escala» vinculado al hacer colectivo de reclamos que cuestiona no solo las actividades nucleares en Córdoba sino también en el territorio nacional. En sintonía con lo que McAdam y Schaffer Boudet (2012) han identificado en múltiples resistencias a proyectos energéticos durante el nuevo milenio en los Estados Unidos, es posible afirmar que las acciones de resistencia a la producción de nucleoelectricidad en Córdoba se habían desplazado de protestas caracterizadas como 
NIMBY a resistencias pasible de ser interpretadas en términos de NIABY (Not In Anybody Backyard); es decir, «No en ningún patio trasero».

Ahora bien, hacia mediados de 2013, los planes de Dioxitek para relocalizarse seguían en pie y contaban con la aprobación de los municipios de Río Tercero y Embalse para que pudieran comenzar con los estudios de prefactibilidad. Sin embargo, el conflicto ganó terreno en la esfera pública y se registró un impulso de la participación ciudadana en la discusión acerca de la aceptación o no de la planta productora de dióxido de uranio en los territorios municipales. Además de conceptualizar a Dioxitek como una instalación riesgosa para el ambiente y la salud de la población, organizaciones ambientalistas y vecinos de Río Tercero buscaron orientar la controversia hacia la discusión del artículo 55 de la carta orgánica. Así, las advertencias públicas de FUNAM, Greenpeace y la Asamblea Permanente por los Derechos Humanos de Río Tercero alertaron a funcionarios del poder judicial que, actuando de oficio, presentaron un recurso de amparo. Según el asesor letrado de Menores de los Tribunales Provinciales en Río Tercero, Alfredo Brouwer de Koning, la ciudad había «vedado la posibilidad de la radicación y funcionamiento de este tipo de industrias en particular y de emprendimientos específicos (centrales nucleares, reservorios, etcétera)» («Plantean amparo contra posible radicación de Dioxitek en Río Tercero», 2013).

Ante la intervención de la justicia y frente a la resistencia pública, Dioxitek ya barajaba otras posibilidades para su traslado. Hacia fines de junio de 2013 las intenciones de una relocalización en la provincia de Córdoba perdían terreno y la provincia de Formosa, ubicada en el extremo noreste de la Argentina y limítrofe con Paraguay, comenzaba a sonar con fuerza como posible destino ${ }^{17}$. Los proyectos de construcción de un Polo Industrial, al que se sumaría una Nueva Planta Productora de Dióxido de Uranio (NPU) que reemplazaría a la ubicada en Córdoba, tomaron estado público en 2014 e impulsaron resistencias tanto en Formosa como en sus provincias limítrofes y la República de Paraguay (Piaz, 2020).

Mientras tanto, el acuerdo que amparaba el funcionamiento de la planta en la capital cordobesa se acercaba a su fin. Vencido los plazos establecidos, en noviembre de 2014 se concretó una nueva clausura de Dioxitek que se extendería en el tiempo, pero que resultaría también provisional. Tras negociaciones varias, la municipalidad y la empresa volvieron a acordar en enero de 2017 un nuevo permiso provisorio para continuar con la producción de dióxido de uranio. 


\section{Discusión y consideraciones finales}

En este trabajo se describieron y analizaron acciones de resistencia al proceso productivo de la nucleoelectricidad que emergieron en la provincia de Córdoba desde los años ochenta hasta la actualidad. Si bien no se identificaron resistencias pasibles de ser consideradas masivas, en consonancia con lo registrado en relación con otras protestas antinucleares que se sucedieron en el país, se desprende de la descripción y el análisis del caso de estudio la existencia de reiterados cuestionamientos a esta tecnología que han promovido impactos varios sobre el proceso productivo de la nucleoelectricidad y el hacer colectivo de reclamos. En este sentido, nos interesa prestar especial atención a consecuencias y resultados que se encuentran vinculados con: i) impactos en procesos tecnológicos; ii) la consolidación de argumentos antinucleares y el impulso de legislaciones orientadas hacia el rechazo a la producción de nucleoelectricidad; y iii) la emergencia de una coalición de actores antinucleares que potenció la visibilidad y magnitud de las protestas en la esfera pública.

En primer lugar, en relación con los impactos de la resistencia en procesos tecnológicos, se ha señalado que en el marco de cuestionamientos públicos y acciones de protesta la CNEA contempló el cese de actividades extractivas durante la década del ochenta en el CMFLG y la no reactivación del proyecto durante el nuevo milenio; la elaboración de acuerdos (entre la CNEA y el municipio de la capital cordobesa) que señalaban la necesidad de remediar pasivos ambientales en el CFC y relocalizar la planta productora de dióxido de uranio; y la existencia de múltiples negativas -tanto en la provincia de Córdoba como en Mendoza y La Rioja- para avanzar hacia la construcción de la NPU bajo la órbita de Dioxitek. Asimismo, se desprende del análisis que se trata de resistencias que los activistas antinucleares han caracterizado en términos de victorias o como procesos exitosos, en tanto han logrado producir resultados (ver Giugni, 1998; Giugni y Grasso, 2015) que se corresponden con sus principales reclamos. Sin embargo, consideramos pertinente explicitar también que la resistencia no emerge en un espacio de vacío sociopolítico, sino que se da en el marco de relaciones con eventos y acciones exteriores -por ejemplo, caída del precio del uranio en escenario internacional, cambios en las normativas referentes a la organización territorial, aumento de la visibilidad de la cuestión ambiental en la esfera pública- que también han impactado en la toma de decisiones, impulsado cambios e incluso promovido el cese de las actividades mencionadas que forman parte del proceso productivo de la nucleoelectricidad. En este escenario, si bien no se plantea la existencia de relaciones unívocas, de tipo causa-consecuencia directa, se considera la 
conformación de un vínculo indisociable entre las acciones de resistencia a la tecnología nuclear y los procesos descriptos y analizados.

En segundo lugar, se observa que tras reiteradas protestas contra la minería de uranio, actividades realizadas en el CFC o en la planta de Embalse, se avanzó hacia la consolidación de la percepción de las actividades nucleares como riesgosas para la salud y el ambiente, principalmente entre vecinos de las instalaciones resistidas y miembros de organizaciones ambientalistas. En este sentido, se observa que los reclamos y acciones de protesta registrados durante las últimas tres décadas no solo se centraron en las actividades resistidas sino que plantearon un posicionamiento crítico hacia el proceso productivo de la nucleoelectricidad. Según activistas antinucleares, que se apoyan en los principales argumentos sostenidos desde el ambientalismo en el escenario global contra los usos de esta tecnología para producir energía (Martin, 2007; Bauer, 2015), este proceso debería ser considerado como riesgoso, costoso y contaminante. Tal caracterización se explicita, por ejemplo, en el proyecto de ley presentado en la Legislatura provincial a fines de abril de 2013. Allí, miembros de la Coalición Córdoba No Nuclear expresaron su propuesta para avanzar hacia una transición energética. De acuerdo con los impulsores del proyecto, se debería abandonar una matriz energética -caracterizada como «fósil y nuclear», pese a que la nucleoelectricidad representa en Argentina un aporte anual del orden del $5 \%$ - en pos de un modelo que privilegie energías renovables, como la eólica o la solar. Más específicamente, en relación con las actividades nucleares en Córdoba, el proyecto de ley destaca la prohibición de la minería de uranio en territorio provincial (dado que la ley n. ${ }^{\circ} 9.526$ de 2008 establece la prohibición de la minería metalífera a cielo abierto); la existencia de una normativa de ordenamiento territorial que estableció la prohibición de funcionamiento de la planta e impulsó la búsqueda de nuevos destinos para Dioxitek; y la existencia de más de una veintena de municipios que han sido declarados como «zonas no nucleares». En cuanto a los artículos específicos, se observa que estos capitalizan y recuperan discusiones pasadas, poniendo en escena reclamos y tópicos que han sido ejes de otras controversias sobre la tecnología nuclear, como el manejo y la disposición final de combustibles gastados y residuos radioactivos $\underline{\underline{18}}$. En otro orden de ideas, la elaboración de este proyecto forma parte también de un proceso que Merlinsky (2013: 49) caracteriza en términos de productividad jurídica de los conflictos ambientales, en tanto busca impulsar la «construcción de nueva legislación y la actualización de leyes que empiezan a tener aplicación -y de ese modo ganar existencia social-». Si bien el proyecto finalmente no prosperó, formó parte de los mecanismos de «protección social», según la caracterización que propone 
Montenegro (2009), implementados por los actores resistentes, y contribuyó con procesos de visibilización de la resistencia al desarrollo nuclear en la provincia de Córdoba.

En tercer lugar, se ha identificado la reemergencia de acciones de protesta y cuestionamientos públicos al desarrollo nuclear en Córdoba tras el accidente ocurrido en Fukushima en 2011, en sintonía con lo registrado en el escenario internacional. Como señalan Hindmarsh y Priestley (2016), si bien los efectos más inmediatos del accidente se vieron reflejados en la reconsideración de políticas y medidas de seguridad de las centrales nucleares, el «desastre» de Fukushima ha impactado también en el surgimiento de un renovado escrutinio internacional de opciones energéticas para un futuro sustentable en el largo plazo, tanto entre el público en general como entre diversos gobiernos que han optado y/o considerado la opción por la producción de nucleoelectricidad. En este sentido, nos interesa señalar que las discusiones en torno a los pedidos de cierre y/o relocalización de Dioxitek -relacionados con reclamos más extensivos que se vinculaban con demandas por el cierre de la central nuclear de Embalse, la no reapertura de sitios explotables (asociados principalmente a la minería de uranio) y el saneamiento de pasivos ambientales vinculados con estas actividades en el CMFLG y en el CMFC- reimpulsaron la discusión por el desarrollo nuclear, la cuestión ambiental y contribuyeron con la conformación de una coalición de actores antinucleares. Siguiendo a Van Dyke y MacCammon (2010: XIV), que definen la noción de coalición a partir de la existencia de «dos o más organizaciones de movimiento social que trabajan en conjunto en busca de objetivos comunes», se observa la conformación de la coalición Córdoba No Nuclear, que logró articular el trabajo conjunto de diversas organizaciones ambientalistas con la intención de potenciar e impulsar protestas antinucleares en Córdoba y otros puntos del territorio nacional $\underline{\underline{19}}$. Asimismo, integrantes de esta coalición impulsaron la participación conjunta -aunque manteniendo estructuras organizacionales diferenciadas- de actores como Greenpeace, la Fundación CEDHA, miembros del portal de medio ambiente y ecología Eco-Sitio, Los Verdes-FEP, la Fundación Tierravida, la Fundación Holderín, FUNDEPS, Conciencia Solidaria, entre otros de relevancia. Una mención especial merece la FUNAM, histórica organización ambientalista y antinuclear, en tanto no formó parte de la coalición ni adhirió explícitamente a esta. Sin embargo, sí participó activamente de múltiples acciones de protestas que se llevaron a cabo en el territorio provincial y en el resto del país junto con otras organizaciones de la RENACE (como por ejemplo BIOS y el $\mathrm{MACH}$ ), impulsando trabajos coordinados y mecanismos de difusión de la resistencia antinuclear. 
Para finalizar, consideramos que este trabajo discute con aquellas posturas, sostenidas principalmente por integrantes de los sectores promotores de la producción de nucleoelectricidad, que consideran que la resistencia a la tecnología nuclear en Argentina puede ser considerada como un fenómeno menor y responde a impulsos contingentes y particulares. Contrariamente a lo que subyace a esta caracterización, que tiende a minimizar e incluso invisibilizar la resistencia antinuclear, se desprende de la descripción y al análisis del caso Córdoba -en sintonía con lo sugerido en trabajos previos (ver Piaz, 2015)- que las acciones de resistencia a la tecnología nuclear no han sido fenómenos aislados ni emergieron en el marco de la creciente conflictividad ambiental que se registró en el país y la región desde comienzos del nuevo milenio. Al contrario, es posible afirmar que se han desarrollado durante más de tres décadas, han contado con diversos niveles de participación ciudadana y visibilidad en la esfera pública y producido impactos varios. Al respecto, quisiéramos hacer una última aclaración. Por un lado, si bien los impactos en procesos tecnológicos no han alcanzado el resultado de máxima identificado por actores antinucleares -es decir, el cese todas las actividades vinculadas con la producción de nucleoelectricidad-, sí se alcanzaron objetivos que han fortalecido la continuidad de la lucha. Por otro lado, la continuidad de la resistencia y la participación de un reducido número de actores antinucleares -pero con un alto grado de compromiso, lo que se observa, por ejemplo, en la persistencia de un trabajo sostenido por más de treinta años- en la provincia de Córdoba han contribuido tanto con el proceso de consolidación de un discurso que ha sido caracterizado en términos de antinuclear (Piaz, 2019) como con la conformación de la coalición de actores antinucleares que participaron de protestas en la provincia de Córdoba y en el resto del país. Asimismo, consideramos posible establecer relaciones entre la histórica resistencia que se llevó a cabo en Córdoba con las más recientes protestas que emergieron en 2017 en contra de la construcción de una central nuclear en la provincia de Río Negro, y que resultaron clave para que el gobernador de la provincia patagónica desestimara el proyecto e impulsara una ley que prohíbe la instalación de este tipo de industrias en su jurisdicción. En términos más generales, consideramos que este trabajo posibilita pensar que la resistencia antinuclear en Córdoba ha contribuido -junto con otras múltiples resistencias que se desarrollaron en el país desde los años ochenta hasta la actualidad-con la reciente conformación del Movimiento Antinuclear de la República Argentina (MARA). 


\section{Notas}

1. La producción de energía en Argentina depende principalmente de la generación térmica, con predominancia del gas natural y el petróleo (en julio de 2019 aportaron un $64 \%$ y $25 \%$ respectivamente). Por su parte, la nucleoelectricidad aportó el $6 \%$, mientras que la energía eólica representó el $5 \%$ restante (Compañía Administradora del Mercado Eléctrico, 2019). « volver

2. La Red Nacional de Acción Ecologista (RENACE) es un colectivo que agrupa desde 1984 a múltiples actores interesados en problemáticas socioambientales. « VOLVER

3. Se trata de un sitio utilizado para la disposición de sobrantes de minerales procesados, popularmente conocido como «el chichón» del Alta Córdoba. Allí se encuentran depositados «36.000 metros cúbicos de material (57.600 toneladas), compuesto por colas de mineral» (CNEA, $\mathrm{s} / \mathrm{f})$ que conforman una elevación de aproximadamente cinco metros cubierta con una capa de suelo que posibilitó el crecimiento de vegetación en la superficie. Estas colas de mineral, es decir, de material sobrante de procedimientos, se almacenaron en el lugar durante desde comienzos de las actividades del CFC en los años cincuenta hasta 1979, dejando un pasivo ambiental públicamente reconocido por la CNEA. « VOLVER

4. En el marco de las resistencias mencionadas, pero también de una caída del precio internacional del uranio y de que, según Plaza (2003), la empresa contratista no cumpliera con la producción estipulada por contrato. « VOLVER
5. De acuerdo con una lectura retrospectiva que las autoridades de Dioxitek hacen de la situación: «En este marco [a mediados de los años ochenta] la municipalidad de Córdoba comienza la aplicación de la ordenanza de ordenamiento territorial y en la caracterización de las zonas de la ciudad de Córdoba. En el barrio de alta Córdoba, no está permitido de acuerdo a la ordenanza este tipo de industrias» (Dioxitek, 2014, párr. 10). « VOLveR

6. Otras de las opciones barajadas fueron San Rafael, Mendoza (donde funcionó Sierra Pintada), y la zona de Los Gigantes, donde funcionó la minera en Córdoba. « VOLVER

7. Entre estas se destacó el trabajo realizado por la Multisectorial del Sur por un Desarrollo Sustentable. « volver

8. Proceso que comenzaría recién tras la audiencia pública celebrada en enero de 2019. Por aquel entonces, el Gobierno de la Provincia de Mendoza informaba que «Después de 30 años, se iniciará el Saneamiento Ambiental de Sierra Pintada en San Rafael». « volver

9. Entre los que se encuentran las protestas contra actividades extractivas -como la megaminería a cielo abierto y la obtención de hidrocarburos mediante fractura hidráulica (fracking); las manifestaciones contra los considerados usos inconvenientes e indeseados del suelo, vinculados con la instalación de industrias con potencial contaminante (como el caso del rechazo a la instalación de plantas productoras de pasta de celulosa a la vera del Río Uru- 
guay); reclamos referentes al saneamiento y la recomposición ambiental de la cuenca Matanza-Riachuelo; o la resistencia a modelos de producción basados en organismos genéticamente modificado (OGM) -en particular, la soja- $y$ a las consecuentes aspersiones con glifosato en inmediaciones de zonas cultivadas. « VOLVER

10. De manera análoga a lo ocurrido luego de accidentes como los registrados en Three Mile Island y Chernóbil, el de Fukushima impactó en la reconfiguración del escenario nuclear mundial con implicancias varias para las políticas públicas y el desarrollo de esta tecnología (Hindmarsh y Priestley, 2016). En este escenario, la discusión por la producción de nucleoelectricidad dista de estar saldada. De hecho, de acuerdo con la Fundación del Archivo Nacional de Energía Nuclear de Holanda (LAKA), aunque se han registrado respuestas de magnitudes disímiles en el mundo, es posible afirmar que en países como Alemania, India, Italia, España, Suiza, Taiwán y los Estados Unidos «las acciones de resistencia en contra de la tecnología nuclear han aumentado claramente» (LAKA, párr. 10, traducción propia). « volveR

11. Asimismo, apoyaban también la iniciativa el Movimiento Antinuclear Zárate-Campana, y las organizaciones Paraguay No Nuclear, Uruguay No Nuclear y la Asociación Civil Ecosistemas Argentinos. Si bien FUNAM no adhirió formalmente a esta campaña, participó activamente de la lucha antinuclear en Córdoba y en el país. « volver

12. En relación con las clausuras registradas, desde la Subsecretaría de Ambiente de la Municipalidad, el Entrevistado B expli- có: «La planta no cuenta con uso de suelo aprobado por una cuestión lógica. Ese espacio institucional que heredó, hoy por hoy, ha quedado tan inserto dentro de la trama urbana que ya requería empezar a tomar algunas decisiones de traslado» (entrevista personal, 27 de mayo de 2016). « VOLVER

13. La decisión de una eventual mudanza se encontraba en sintonía con los mencionados planes de la CNEA de reactivar la minería de uranio en diversos puntos estratégicos del país. « VOLVER

14. Al respecto, la Asamblea declaraba que «como fruto de la lucha del pueblo y la resistencia a la megaminería, conseguimos después de varios meses la sanción de una ordenanza, la n. ${ }^{\circ}$ 4.930» (Asamblea Riojana Capital, 2013: párr. 8). « volveR

15. El artículo establece que «No pueden radicarse en el mismo [territorio de la jurisdicción municipal] centrales, reservorios, basureros o industrias nucleares. Se prohíbe la manipulación, uso y/o transporte de sustancias o insumos nucleares o afines, que pongan en peligro la vida, la salud de los habitantes y el ecosistema». « volver

16. De manera análoga a lo que ocurriría en la provincia de Formosa, donde se avanza en la construcción de la NPU (ver Piaz, en prensa). « vOLVER

17. Además de presentarse como una solución a la disputa por localización de la planta en su territorio de origen, con esta medida se buscaba fortalecer un camino que había comenzado a consolidarse desde 2010 tras la firma de un acuerdo que promovía la construcción en la provincia norteña un reactor tipo CARMEM; es decir, 
un reactor nuclear de baja potencia -de diseño nacional- con capacidad para generar energía en el orden de los $150 \mathrm{Mw}$. Actualmente la CNEA avanza en la construcción de un prototipo en Lima, provincia de Buenos Aires, en el mismo predio donde funcionan Atucha I y Atucha II. « volver

18. Mientras que el artículo 1 sostiene que «Se declara a la Provincia de Córdoba territorio no nuclear. Se prohíbe en todo el territorio provincial la generación de energía eléctrica en base a reactores nucleares», el artículo 2 -retomando las discusiones acerca de los residuos radiactivos o elementos combustibles gastados que se planteara en el caso INVAP- establece la conceptualización del «combustible nuclear agotado» como un residuo radiactivo. El artículo 3, en sintonía con las acciones de resistencia en contra de la creación del repositorio final de residuos radiactivos en Gastre, establece la prohibición «en todo el territorio de la Provincia de Córdoba [de] la construcción y operación de repositorios para el almacenamiento, transitorio o definitivo, de residuos radiactivos», dejándose asentados en el artículo 4 como excepciones aquellos residuos generados por los reactores de potencia emplazados en la provincia, aquellos provenientes de actividades pasadas -vinculadas a la minería de uranio y/o procesamiento del mismo-, y aquellos resultantes de actividades vinculadas a los usos medicinales de la tecnología nuclear. Los siguientes artículos buscan establecer, entre otras cuestiones de relevancia, la prohibición del transporte de residuos radioactivos por el territorio provincial y la exigencia del cese de actividades de Dioxitek una vez finalizados los plazos acordados con la Municipalidad de Córdoba para su funcionamiento. La autoridad de aplicación propuesta por la ley es el Ministerio de Agua, Ambiente y Energía de la Provincia de Córdoba, que debería actuar en consonancia con las responsabilidades competentes a la CNEA y la ARN. « VOLVER

19. Como en la controversia que emergió en torno a la construcción de la NPU en Formosa (ver Piaz, en prensa), o en las protestas que se realizaron entre 2012 y 2013 en la Ciudad Autónoma de Buenos Aires y frente las centrales nucleares Atucha I y Atucha II. « volveR 


\section{Referencias bibliográficas}

ASAMBLEA RIOJANA CAPITAL (2013). Situación en La Rioja departamento capital. Disponible en: http://asambleariojanacapital.blogspot.com.ar [26 de septiembre de 2016].

BAUER, M. (2015). Atoms, Bytes and Genes. Public Resistance and Techno-Scientific Responses. Nueva York: Routledge.

BREYMAN, S.; Campbell, N.; Eubanks, V. y Kinchy, A. (2017). STS and social movements: Past and futures. En: Felt, U. y otros (eds.), The Handbook of Science and Technology Studies. Fourth Edition 289-317. Cambridge, Estados Unidos: The MIT Press.

CARRUTHERS, D. (2008). Environmental Justice in Latin America: Problems, Promise, and Practice. Cambridge y Londres: The MIT Press.

COMISIÓN NACIONAL DE ENERGÍA ATÓMICA (s/f). Sitios en Córdoba. Disponible en: http:// www.cnea.gov.ar/remediacion-sitios-cordoba [22 de junio de 2016]

COMPAÑÍA ADMINISTRADORA DEL MERCADO ELÉCTRICO (2019). Informe mensual. Principales variables del mes. Julio de 2019. Disponible en: http://ageera.com.ar/ wp-content/uploads/2019/09/Informe-Mensual-CAMMESA-julio-2019.pdf [12 de septiembre de 2019].

CÓRDOBA FRENTE A LOS PELIGROS DE LA ENERGÍA NUCLEAR (2011). Argentina Indymedia. Disponible en: http://argentina.indymedia. org/news/2011/05/778670.php [4 de mayo de 2016].

DICHDJ,, A. (2018). «La Epopeya Antinuclear». El Caso de Gastre (Provincia de Chubut) Como Sumidero Radiactivo Frustrado en Argentina (1980-1990). HALAC.
Historia Ambiental Latinoamericana $y$ Caribeña, 8(2), 152-179. DOI: https://doi.org/10.32991/2237-2717.2018v8i2.p152-179

DiOXITEK S.A. (2014). Comunicado de Prensa PRES N³/14. Disponible en: http:// www.normamorandini.com.ar/wp-content/ uploads/2013/12/RESPUESTA-DE-DIOXITEK-SA.pdf [20 de marzo de 2016].

El tRASLAdo de la PLANTA dE URANIO DIVIDE A DESPEÑAdEROS (1999). La Voz. Disponible en: http://buscador.lavoz.com.ar/intervoz/99/02/07/ig n2.htm [25 de septiembre de 2019].

FORNI, P. y Leite, V. (2006). El desarrollo y legitimación de las organizaciones del tercer sector en la Argentina. Hacia la definición de un isomorfismo periférico. Sociologias, 8(16), 216-249.

GIUGNI, M. (1998). Was it worth the effort? The outcomes and consequences of social movements. Annual Review of Sociology, 98, 371-393.

HuRTADO, D. (2014). El sueño de la Argentina atómica. Política, tecnología nuclear y desarrollo nacional (1945-2006). Buenos Aires: Edhasa.

LANDELIJK KERNENERGIEARCHIEF [Archivo Nacional de Energía Nuclear] (LAKA). Responses after Chernobyl and Fukushima. Comparative analysis of German and The Netherlands as amplified examples. Nuclear Monitor, 743. Disponible en: http:// laka.org/info/publicaties/2012-chernobyl-fukushima.pdf [26 de septiembre de 2019].

LOS VERDES (2014). Los mil intentos de Dioxitek o el rechazo a la industria nuclear. 
Disponible en: http://www.losverdes.org.ar/ wp-content/uploads/2014/07/LOS-MIL-INTENTOS-DE-DIOXITEK.pdf [21 de julio de 2016].

HINDMARSH, R. y Priestley, R. (eds.) (2016). The

Fukushima Effect. A New Geopolitical Terrain. Nueva York: Routledge.

MARTIN, B. (2007). Opposing nuclear power: past and present. Social Alternatives, vol. 26, n. ${ }^{\circ}$ 2, pp. 43-47. Disponible en: https://documents.uow.edu.au/ /bmartin/ pubs/07sa.html [21 de mayo de 2020].

MARTIN, H. (2015). Reflexiones sobre las actividades de la Comisión Nacional de Energía Atómica en Córdoba. Período: 30 de Agosto de 1952 - 7 de Noviembre 2012. Disponible en: http://www.cba.gov. ar/wp-content/4p96humuzp/2016/01/REFLEXIONESsobreACTIVIDADESdeCNEAenCORDOBA.pdf [22 de julio de 2016].

MARTínez Demarco, S. (2012). Minería del Uranio en Sierra Pintada: Conocimiento, Comunidades Epistémicas y Desarrollo. VII Jornadas de Sociología, Universidad Nacional de General Sarmiento, Buenos Aires, Argentina. Disponible en: http://www.ungs. edu.ar/ms ici/wp-content/uploads/2012/11/ Martinez-Demarco-GT5.pdf [26 de septiembre de 2019].

McAdam, D.; Tarrow, S. y Tilly, C. (2007). Comparative perspectives on contentious politics. En Lichbach, M. y Zuckerman, A. (eds.), Comparative Politics: Rationality, Culture, and Structure. Advancing Theory in Comparative Politics. Cambridge: Cambridge University Press. Disponible en: http:// socialsciences.cornell.edu/wp-content/ uploads/2013/06/McAdamTarrowTilly07.pdf [26 de septiembre de 2019].
MERLINSKY, G. (2013). Política, derechos y justicia ambiental. El conflicto del Riachuelo. Buenos Aires: Fondo de Cultura Económica. MONGI, C. y Martínez, M. (2013). Dioxitek: Ambiente exigirá que haya estudios de impacto. La Voz. Disponible en: http://www.lavoz. com.ar/embalse/dioxitek-ambiente-exigira-que-haya-estudios-impacto [26 de septiembre de 2019].

MONTENEGRO, R. (2009). Argentina's irrational nuclear programme and citizen's opposition. En Mez, L. y otros (comps.), Perspectives on Energy Policy and the Role of Nuclear Power. Essex: Multi-Science Publishing Co.

NELKIN, D. (1995). Science controversies: The dinamics of public disputes in the United States. En Jasanoff, S. y otros (eds.), The Handbook of Science and Technology Studies, 444-456. California: Sage.

OTTINGER, G.; Bararndiarán, J. y Kimura, H. (2017). Environmental justice: Knowledge, technology, and expertise. En Felt, U. y otros (eds.), The Handbook of Science and Technology Studies. Fourth Edition, 289 317. Cambridge, Estados Unidos: The MIT Press.

PIAZ, A. (2015). Acciones de resistencia a la tecnología nuclear en Argentina: mapeando el terreno. REDES, Revista de Estudios Sociales de la Ciencia y la Tecnología, 21(41), 111-140.

PIAZ, A. (en prensa). Riesgo, tecnología nuclear y acciones de resistencia en Formosa: la controversia en torno al proyecto CAREM y la NPUO2. CTS, Revista Iberoamericana de Ciencia, Tecnología y Sociedad.

PIAZ, A. y Vara, A. M. (2013). Tecnología nuclear, percepción de riesgo y debate entre 
expertos: la movilización por las napas de agua en Ezeiza. En Tula Molina, F. y Vara, A. M. (comps.), Riesgo, política y alternativas tecnológicas. Entre la regulación y la discusión pública, 359-415. Buenos Aires: Prometeo.

PLAZA, H. (2003). La industria del uranio en Argentina. Seguridad Radiológica, 22, 16-21.

PISANI, S. (1997). Admiten que hay restos nucleares en la ciudad de Córdoba. La Nación. Disponible en: https://www.lanacion.com. ar/sociedad/admiten-que-hay-restos-nucleares-en-la-ciudad-de-cordoba-nid66705 [26 de septiembre de 2019].

PLANTEAN AMPARO CONTRA POSIBLE RADICACIÓN DE DIOXITEK EN Río TERCERO (2013). La Mañana. Disponible en: http://www.lmcordoba.com.ar/nota/130440 plantean-amparo-contra-posible-radicacion-de-dioxitek-en-rio-tercero [8 de agosto de 2016].

POLÉMICA POR POSIBLE TRASLADO DE UNA PLANtA ATÓmICA A SAN RAFAel (2004). Los Andes. Disponible en: http://archivo.losandes.com. ar/notas $/ 2004 / 7 / 20 /$ sociedad-117900.asp [14 de abril de 2016].

Rodríguez PARDO, J. (2006). En la Patagonia NO. Crónica de la epopeya antinuclear de Gastre, veinte años que impidieron el ba- surero atómico en Chubut. El Bolsón: Proyecto Lemu-Grupo Amigos del Libro.

Svampa, M. (2012). Cambio de época. Movimientos sociales y poder político. Buenos Aires: Siglo XXI editores.

TARROW, S. (2011). Power in Movement. Social Movements and Contentious Politics. Revised and Updated Third Edition. Nueva York: Cambridge University Press.

VAN DYKE, N. y McCammon, H. (2010). Introducción: Social movement coalition formation. En Strategic Alliances, Coalition Building and Social Movements, XI-XXVIII. Minneapolis-Londres: University of Minnesota Press.

\section{Entrevistas citadas}

ENTREVISTADO A: Ambientalista, miembro de FUNAM y activista antinuclear. Entrevista realizada en la ciudad de Córdoba, el 21 de mayo de 2015.

ENTREVISTADO B: Miembro de la Subsecretaría de Ambiente de la Municipalidad de Córdoba. Entrevista realizada el 27 de mayo de 2016.

ENTREVISTADO C: Miembro de la agrupación Los Verdes. Entrevista realizada mediante correspondencia personal el 21 de mayo de 2015. 